\title{
Procesos cognitivos en daño cerebral por infección
}

\author{
Cognitive processes in infection brain damage
}

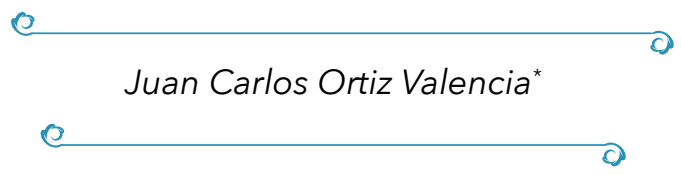

Recibido: $15.05 .2019 \bullet$ Arbitrado: 11.06.2019•

Aprobado: 25.06.2019

Psicólogo UNAD, Esp. Adicciones Universidad Católica Luis Amigó, Maestría en Neuropsicología, Universidad de San Buenaventura, Colombia, ORCID: 0000-0001-8360-1693, jortiz_valencia@hotmail.com. Investigador independiente.

\section{Resumen}

Concepto de procesos cognitivos se refiere a las capacidades intelectuales propias del ser humano, a la interacción mente cuerpo y/o mente entorno, para entender el mundo desde la interacción recíproca y constante de percepción, lenguaje, toma de decisiones, memoria, y emociones. Pero la misma fragilidad humana nos hace proclives a padecer tanto el deterioro normal del envejecimiento, como el causado por agentes virales, bacterianos, parasitarios y fúngicos que alteran sin duda tales procesos. Objetivo: Conocer las características de la valoración de procesos cognitivos cuando hay daño o deterioro cerebral por infección. Metodología: Revisión descriptiva a través de EBSCO, NATURE - International Weekly Journal of Science-, OXFORD academic, PsycARTICLES American Psychological Asociation, Science Direct (Journals), SCOPUS, Google Scholar, Redalyc.Org. y Scielo para información no anterior al año 2014. Resultados: Se encontró que si bien los diferentes agentes patógenos pueden alterar la estructura celular del cerebro, y en consecuencia sus procesos cognitivos, este presenta una sensibilidad elevada a la inflamación provocada por virus; sin embargo, no se sabe mucho de cuanto podría 
deteriorarse alguna función cognitiva frente a infecciones virales comunes y aparentemente inocuas. Conclusiones: Se hace importante poder discernir el cuidado de las diferentes funciones cognitivas, tanto desde prácticas de estilos de vida saludable o prevención primaria como desde el uso de la tecnología computarizada que permite la optimización de la valoración neuropsicológica para las funciones cognitivas.

Palabras clave: Evaluación, Procesos Cognitivos, Infección Cerebral, Daño Cerebral, Deterioro Cognitivo.

\section{Abstract}

Cognitive processes let thinking about intellectual human abilities, interacting bodymind, and/or environment- mind, to understand the world from reciprocal and constant perception, speech, making decisions, memory, and emotions. But human fragility make us susceptible to suffer, both the normal aging impairment and caused by viral, bacterial, parasitic and fungal agents that alter such processes. Objective: To know the characteristics of cognitive processes assessment when there is brain damage or deterioration by infection. Methodology: Descriptive review trough data bases like EBSCO, NATURE - International Weekly Journal of Science-, OXFORD academic, PsycARTICLES American Psychological Asociation, Science Direct (Journals), SCOPUS, Google Scholar, Redalyc.Org, y Scielo for information after to 2014. Results: Was found it that while different pathogens can alter de cellular estructure of the brain, and consequently their cognitive processes, this have a high sensibility to inflammation caused by virus; but nevertheless, there is no much known about how much cognitive function could be impaired and its response to common and aparently innocuous viral infection. Conclusions: It becomes important to be able to discern the care of different cognitive functions, both from healthy style life or primary prevention and from computarized technology using that allows the optimization of neuropsychological assessment for cognitive functions.

Keywords: Evaluation, Cognitive Processes, Brain Infection, Cerebral Damage, Cognitive Impairment.

\section{Introducción}

Al hablar de procesos cognitivos, se alude a algunas de las capacidades intelectuales propias del ser humano, desde la interacción mente cuerpo y/o mente entorno, y desde la interacción recíproca y constante de la percepción, el lenguaje, la toma de decisiones, la memoria, y las emociones, en el marco de patrones de activación y elementos informativos que operan simultánea- 
mente y facilitan la construcción constante de significados del mundo y de la vida. Sin embargo, debido a su misma naturaleza, el ser humano es proclive, tanto desde el deterioro normal por envejecimiento, como por agentes virales, bacterianos, parasitarios y fúngicos, a que estas construcciones se vean suspendidas o paralizadas de manera termporal en ocasiones y en otras de manera permanente.

En este sentido se hace importante la revisión sistémica de los procesos cognitivos en daño cerebral en el marco de procesos infecciosos por agentes diversos, con el fin de comprender la magnitud de los cambios estructurales posibles desde patologías comunes y aparentemente inocuas hasta otras mas complejas como la encefalitis japonesa, HIV, malaria, cuyos sobrevivientes cuentan desde sus componentes cerebrales y mentales una historia de cambio, de fragilidad pero de capacidad compensatoria y adaptativa. Estas aproximaciones, permiten ampliar el panorama de todo aquel que haya decidido desde las neurociencias aportar al beneficio de la humanidad y a mejorar procesos de intervención y rehabilitación desde la visión de diferentes autores/ investigadores alrededor del mundo.

Tratar entonces de responder a cuales son las características de los procesos cognitivos en daño cerebral por infección, parece ser una tarea bastante amplia y ardua, con lo cual lo presentado en este artículo podría tomarse como una aproximación al ejercicio de la revisión científica de un tema que podría y seguramente ha tomado, años de investigación a quienes han decidido indagar al respecto. Sin embargo, es posible intentar comprender, grosso modo, los efectos de procesos infecciosos de diferente etiología en el cerebro y en consecuencia en la evolución neurocognitiva de los individuos que las han padecido.

De esta manera, el objetivo de esta revisión se ha centrado en conocer las características de la evaluación de procesos cognitivos cuando hay daño o deterioro cerebral por infección independiente del agente causal.

\section{Metodología}

Para la realización de este trabajo de revisión descriptiva, se llevó a cabo la búsqueda bibliográfica a través de bases de datos como: EBSCO, NATURE International Weekly Journal of Science-, OXFORD academic, PsycARTICLES American Psychological Asociation, Science Direct (Journals), SCOPUS; y otras de libre acceso como Google Scholar, Redalyc.Org, y Scielo, mediante las cuales se filtró información no mayor a 5 años (desde 2014 hasta 2019) 
relacionada con procesos cognitivos, evaluación de procesos cognitivos, daño cerebral, infección cerebral y deterioro cognitivo por infección.

La información obtenida fue filtrada desde la metodología propuesta en la Guía Programa de Habilidades en Lectura Crítica CASPe (Cabello, 2003) a través de las tres preguntas básicas de eliminación: 1.¿Existió una comparación con una prueba de referencia adecuada?, 2. ¿Incluyó la muestra un espectro adecuado de pacientes?, y 3.¿Existe una adecuada descripción de la prueba?

Para la organización de la información y posterior redacción, se ha hecho uso de Microsoft Excel, Microsoft Word y Gestor bibliográfico, red social y base de datos Mendeley.

\section{Desarrollo y Discusión}

Al hablar de procesos cognitivos, es posible que se piense en algunas de las capacidades intelectuales propias del ser humano, con relación a la interacción mente cuerpo y/o mente entorno, sin embargo, es importante clarificar esto con el ánimo de evitar imprecisiones conceptuales que opaquen la posibilidad de la relación entre la función mental y algunas afecciones cerebrales inducidas por procesos infecciosos.

Así, desde el aprendizaje y la psicología cognitiva, la complejidad de comprensión de algunos conceptos algebráicos, trigonométricos y la solución de problemas, requiere de estrategias cognitivas que aporten en la posibilidad inductiva o deductiva que requiera determinado ejercicio; es decir, no solo memorizar, sino encontrar la aplicación práctica de la información obtenida; no solo percibir, sino comprender lo percibido. Es entender el mundo desde la interacción recíproca y constante de percepción, lenguaje, toma de decisiones, memoria, emociones; desde los patrones de activación y elementos informativos operando simultáneamente. En en otras palabras, es ser capaz de construir significados desde los diferentes aspectos y manifestaciones del mundo y la vida (Aguado, 2002; Introzzi, Canet-Juric, Montes, López y Mascarello, 2015; Mariángel y Jiménez, 2015; Ravindran, Rani y Priya, 2014; Rivas N, 2008) o, según Vigotsky, es la intervención de elementos psicológicos y simbólicos, los que "modifican radicalmente el proceso de aprendizaje, ...y regular sus propios procesos cognitivos con la ayuda de estos instrumentos culturales" (Vergel, 2014).

Desde una óptica más biológica, se hace alusión a los procesos cognitivos como "puentes" que actúan entre el sistema nervioso y la conducta, lo que los convierte en necesarios casí como los ingredientes en la receta de una cocina 
que se complementan unos a otros para lograr un resultado final o transitorio esperado (Aguado, 2002; Marino, Jaldo, Arias y Palma, 2017). Es así como tienen lugar la capacidad de fijación hacia un estímulo dado, la modulación de la atención (Carmilema y Fajardo, 2018; Guzmán de R, 2013; Luna-Lario, Azcárate-Jiménez, Seijas-Gómez, y Tirapu-Ustárroz, 2015) desde el estímulo propuesto, la comprensión del lenguaje desde sus diferentes presentaciones culturales, la lectura, escritura, construcción y entendimiento semántico y gramatical; en suma las habilidades metacognitivas y predictivas del cerebro en pro de un fin (Fernández, Orozco y Agamennoni, s.f.; Llinás, 2003; Macbeth, Cortada, Razumiejeczyk y López, 2008) cuya regulación, como se ha encontrado, está a cargo de distintas áreas del lóbulo frontal, sobre todo para memoria, lenguaje y función ejecutiva (Chimbo Ch, 2014; Golub, Chase, Batista, y Yu, 2016; Introzzi et al., 2015; Moreno et al., 2016; Silveira, 2016).

Lo anterior, sin duda, tiene gran implicación en situaciones académicas, laborales, familiares, sociales, con lo cual los procesos cognitivos, siendo transversales entre sí mismos, resultan transversales a la vida y cotidianidad humana. Es así, como tienen formación dinámica desde el nacimiento del sujeto, y pasan por estadíos específicos en los que se potencian unas destrezas u otras como la capacidad de planificación (6 - 8 años), el control inhibitorio (12 - 14 años), y otras como la flexibilidad cognitiva, memoria de trabajo, y resolución de problemas (15 - 19 años) (Megías et al., 2015). Ya con el envejecimiento normal, la tendencia es a la disminución en la eficacia de estas capacidades, permitiendo evidenciar una lentificación generalizada de estas alrededor de la cuarta y quinta decadas de la vida (García-Herranz, Díaz-Mardomingo y Peraita, 2014; Marino et al., 2017; Mias, Bastida, Del Boca y Legeren, 2017; Rojo-Mota, Pedrero-Perez, Ruiz-Sánchez, Llanero-Luque y Puerta-Garcia, 2013; Sandoz, Démonet y Fossard, 2014; Solís-Marcos, Castellano-Guerrero, Domínguez Morales y León-Carrión, 2019).

Por lo tanto, es en esta fase de la vida (durante el envejecimiento del adulto mayor) en que podrían presentarse olvidos frecuentes de fechas, eventos o la localización de objetos; dificultad para aprender nueva información, para nombrar palabras dentro de una categoría semántica, entre otras, que podrían compensarse con la memoria cristalizada y la reserva cognitiva de cada sujeto. No obstante, según Ollari (1999), en la mayoría de casos, durante el envejecimiento normal, se conservan funciones de memoria procedimental, praxias, gnosias y lenguaje, pero durante el envejecimiento patológico, el declive de dichas funciones es pronunciado, afectando llamativamente la cotidianidad del sujeto, y muchas veces desencadenando en las conocidas demencias (Alzheimer, Parkinson, Huntington...) con la desgracia de 
identificar sus síntomas casi siempre cuando el progreso de la enfermedad ha llegado a fases muy avanzadas (García-Herranz et al., 2014; Mias et al., 2017; Nimgaonkar et al., 2016; Rojo-Mota et al., 2013; Villalba y Tortajada, 2014).

En este orden de ideas, se hace importante poder discernir el cuidado de las diferentes funciones cognitivas, tanto desde prácticas de estilos de vida saludable o prevención primaria (Portellano, 2005; Solís-Marcos et al., 2019), como desde el uso de la tecnología computarizada que permite la optimización de la valoración neuropsicológica para las funciones cognitivas, no solo dentro de patrones de envejecimiento, pero también en eventos traumáticos y mórbidos que pudiesen afectar tales procesos mentales (Hagler, Brugge y Pavel, 2014). De igual forma, se ha visto que la "comparación normativa multivariable" puede ser mas efectiva en la detección de deterioro cognitivo que los criterios de Frascati para trastornos neurocognitivos asociados a VIH, por ejemplo (Su et al., 2016).

Como bien se mencionó previamente, el deterioro cognitivo puede venir no solo del envejecimiento normal, pero también de daño cerebral por trauma, o por procesos mórbidos, infecciosos o inflamatorios. Con relación a tales procesos infecciosos, se ha establecido la relación que existe entre estos y la capacidad inflamatoria celular como uno de tantos mecanismos de defensa, pero esta actividad inflamatoria, sostenida en el tiempo, bien sea por proceso infeccioso refractario, o por hiperreacción inmunitaria, pone en desventaja a las estructuras adyascentes, para el caso, neuronas y neuroglía. Es así como diversos estudios han evidenciado una correlación entre la inflamación del sistema nervioso central y deterioro cognitivo ligado a enfermedades como Alzheimer, por la hiperproducción de placas amiloideas, autismo, déficit de atención e hiperactividad, y otros de tipo neuropsiquiátrico como esquizofrenia (Fan, Holland, Loxton y Barghouth, 2015; Ferrari et al., 2016; Hamdani et al., 2015; Karachaliou et al., 2016; Lattanzi, Luzzi, Provinciali y Silvestrini, 2014; Licastro, Raschi, Carbone, Porcellini y Caruso, 2015; Takeda, Sato y Morishita, 2014) que se ampliaran mas adelante.

Otros estudios han descrito deficit cognitivos asociados a infección, tales como falta de progreso en el desarrollo y en el habla, síntomas depresivos y demás déficit cognitivos seguramente relacionados con demencia, confusión mental y deterioro cognitivo, deterioro de la fluidez mental de la memoria a corto plazo y de trabajo, y disminución de la velocidad de procesamiento. Específicamente en daño por infección con T. Canis, se ha encontrado pobre rendimiento en lectura, distractibilidad y baja inteligencia en niños de jardín. Luego, Marmor et al. (1987) con baterías neuropsicológicas aplicadas, encontró déficit cognitivos significativos en niños seropositivos para T. Canis, entre 
1 y 15 años de edad; y Nelson, Greene y Ernhart (1996) en niños entre 1 y 4 años, seropositivos para el mismo parásito, observó niveles de inteligencia mas bajos que sus compañeros seronegativos (Fan et al., 2015; Karachaliou et al., 2016; Marmor et al., 1987). En otras investigaciones, se ha hallado relación entre la exposición a Citomegalovirus CMV, Herpes Simple Virus 2 HSV - 2, y Toxoplasma Gondii TOX, en individuos mayores y deterioro cognitivo (Ackermann, van Toorn y Andronikou, 2019; Fan et al., 2015; Gicas et al., 2014; Hung et al., 2018; John et al., 2015; Karachaliou et al., 2016; Licastro et al., 2015; Nimgaonkar et al., 2016; Widmann y Heneka, 2014).

Con relación al deterioro del habla y del lenguaje asociados a infecciones por virus, también han sido reportados en la literatura. Un considerable número de sobrevivientes a encefalitis por herpes simple HSV, presentan con el tiempo déficit cognitivos relacionados con memoria, nominación, conocimiento semántico, funciones ejecutivas y regulación emocional, esto ligado a cambios cerebrales observados en valoraciones neuropsicológicas como perdida de la sustancia gris en lóbulo temporal lateral, tálamo e ínsula izquierda; con un impacto final negativo del sujeto, para interactuar con su entorno social y académico (Hung et al., 2018). Las infecciones por virus en el sistema nervioso central pueden tener efectos deteriorantes para el desarrollo del cerebro, pero el efecto de infecciones comunes menos virulentas no está claro; no obstante, el desarrollo del cerebro es demasiado sensible a la neuroinflamación inducida por virus (Hung et al., 2018; Karachaliou et al., 2016).

Con relación al Toxoplasma Gondii TOX, es importante saber que una infección por este agente puede tener un curso asintomático, no obstante generar deterioro cognitivo, sobre todo a nivel de memoria, que se evidencia en la adultez, alrededor de los 60 años, lo cual genera un gran impacto para la salud pública con consecuencias socioeconómicas considerables (Cohen et al., 2015; Gajewski, Falkenstein, Hengstler y Golka, 2014). Muchas veces estas condiciones sociales precarias, no son la consecuencia, sino también una de las causas para que bajo otras situaciones oportunistas, incluyendo las infecciones, los individuos pierdan capacidades cognitivas, involucionando en un deterioro importante. Asi se ha observado en grupos socialmente marginados, con pocos años de educación, alta dependencia a heroína, alta exposición a infecciones por virus, síntomas negativos severos, y gran amplitud de síntomas neurológicos blandos, quienes mostraron un funcionamiento cognitivo mas pobre que el de otros grupos, sobre todo en desempeños de memoria verbal, atención y flexibilidad mental; adicionalmente, deterioro de la integridad cerebral, mayor exposición viral y mayor uso de alcohol; sin embargo, su capacidad de tomar decisiones, habilidades inhibitorias, y coeficiente 
intelectual, aparecían en límites normales, lo que podría sugerir un "ahorro selectivo" (Gicas et al., 2014; Rivas, 2008).

En procesos infecciosos por HIV, se ha evidenciado en estudios volumétricos, un parénquima cerebral total reducido, expansión del tercer ventrículo y alargamiento del tallo cerebral; de igual forma alteración en la sustancia blanca, y alteraciones microestructurales en el caudado. En otros estudios realizados poco después de la seroconversión, se encontró daño en la corteza frontal, activación de la microglía en ganglios basales, reducción del flujo sanguineo subcortical, y cambios estructurales en materia gris con expansión del tercer ventrículo. También se ha descrito desmielinización y daño axonal con disfunción (Ackermann, van Toorn y Andronikou, 2019; Ragin et al., 2015; Su et al., 2016). El deterioro cognitivo es altamente prevalente en pacientes infectados con HIV, aún a pesar de que la infección parezca adecuadamente controlada por los antirretrovirales (Su et al., 2016). Infecciones en niños por HIV se relacionan con un amplio rango de problemas cognitivos (Ackermann et al., 2019; Cohen et al., 2015; Gicas et al., 2014; Marino et al., 2017; Ragin et al., 2015).

Las infecciones que causan alta morbilidad al sistema nervioso central SNC, generalmente incluyen virus (encefalitis japonesa, rabia, HIV, herpes simple, herpes zoxter, citomegalovirus, dengue, chicungunya), bacterias (tuberculosis, sifilis, meningitis bacteriana, sepsis), fúngicas (meningitis criptocóccica) y parasitos (malaria, neurocisticercosis, neuroschistosomiasis, helmintos transmitidos por el suelo), y afectan a millones de personas, niños y adultos provocando grandes problemas de salud mental, neurológica, cognitiva y comportamental (John et al., 2015). Sin embargo, a veces el manejo podría resultar lesivo, con en el tratamiento para helmintos, el cual por sí mismo podría desencadenar un proceso inflamatorio vascular (vasculitis) provocando infartos en diferentes áreas cerebrales y, en consecuencia, afectación cognitiva según el correlato anatómico dañado (Fan et al., 2015).

\section{Infecciones virales}

Las consecuencias cognitivas desde el grupo de las infecciones de tipo viral, son diferentes según el agente causal y obviamente según el reflejo de los estudios que se hayan llevado a cabo al respecto. De esta manera en el grupo de los Arboviruses (dengue, chicungunya y encefalitis japonesa), solo se tienen información de los sobrevivientes de la encefalitis japonesa, quienes alrededor de 30 a 50\% tienen secuelas neurológicas, psiquiátricas o cognitivas (Spagnolo, Scomazzoni, Fichera, Comi y Volontè, 2014; Zhao et al., 2015). 
Para las infecciones por Rhabdoviruses (rabia), las consecuencias cognitivas son descritas como fatales. En infecciones por Herpesviruses, la consecuencia cognitiva para encefalitis por HSV incluye deterioro de la memoria en un 69\%; para virus Varicela Zoxter VZV los resultados a nivel cognitivo no han sido concluyentes; y en infecciones por citomegalovirus congénito CMV se relaciona con retraso global del desarrollo en el 50\% de los niños afectados. En HIV se ha visto deterioro neurocognitivo asintomático, desorden neurocognitivo leve y demencia asociada. Infecciones por HIV ligada a meningítis criptocóccica, desarrollan un mimetismo con las demencia vascular y otro tipo de demencia reversible; y en encefalitis por Toxoplasma el desenlace cognitivo es la demencia (John et al., 2015). Por su parte, el Poliomavirus, BKP y V, es un oportunista, con gran afinidad por el SNC tanto en niños como en adultos, y su infiltración podría provocar distorsiones en diferentes vías neurales importantes para la atención, con consecuencias como inatención o hiperactividad (Karachaliou et al., 2016).

El Zika virus o ZIKV, puede ocasionar microcefalia en el feto, aunque sus efectos en el crecimiento corporal son altamente desconocidos, pero se sabe que individuos infectados con este virus presentan alteración marcada en el crecimiento y déficits en el aprendizaje espacial y la memoria, con reducción importante de hormonas para el crecimiento durante la fase aguda de la infección (Wu et al., 2018).

Deterioro en el habla y el lenguaje, pese a que podrían ser problemas comunes durante el desarrollo de niños y jóvenes, existen pocos estudios que podrían evidenciar una relación creciente entre dichas alteraciones y los Enterovirus EV, subsecuente a la neuroinflamación secundaria a infecciones de este tipo (Hung et al., 2018)there has been minimal study of the association between common childhood infections (e.g. enterovirus [EV].

\section{Infecciones bacterianas}

En el ámbito de las infecciones bacterianas, se ha observado en la Sepsis, también conocida como endotoxemia o envenenamiento de la sangre, y en la Meningitis Neonatal, deterioro cognitivo (a pesar de los pocos estudios), retraso del desarrollo, dificultades para el aprendizaje, y meningitis. La sepsis per se, produce encefalopatía asociada, con rápida disfunción de la memoria y subsecuentemente confusión y coma. Los sobrevivientes a septicemia podrían desarrollar alto riesgo de deterioro cognitivo a largo plazo y demencia (Widmann y Heneka, 2014). La meningitis bacteriana propiamente dicha, produce en los niños daños relacionados con deterioro cognitivo ligado a bajo 
coeficiente intelectual, limitaciones académicas, disfunción ejecutiva, y en adultos deterioro cognitivo ligado a baja velocidad de procesamiento. Para la meningitis tuberculosa (infección oportunista en HIV), el deterioro cognitivo se ha observado en todas las áreas evaluadas y pobre progreso académico. En la neurosífilis, el deterioro se aprecia a nivel de memoria, orientación y demencia (John et al., 2015).

\section{Infecciones por parásitos}

Desde las infecciones por parásitos, parece ser que la que deja mayores rastros de deterioro corresponde a la Malaria, la cual puede afectar la atención, memoria de trabajo, habilidades visoespaciales, la discriminación somatosensorial, el habla, lenguaje expresivo y receptivo, la función ejecutiva, coordinación motora fina, atención y razonamiento abstracto. La neurocisticercosis afecta la capacidad de nominación, fluidez verbal y la memoria no verbal. En STH por T. Trichiura y A. Lumbricoides, el deterioro se observa en la incapacidad para el aprendizaje, la memoria verbal, y reducida tasa de procesamiento y atención. Por último, la quistosomiasis, sin producir infección de tipo neurológico, si que afecta la memoria verbal y la fluidez verbal (John et al., 2015).

Alrededor del 30\% de los humanos ha tenido infección por parásitos como T. Gondii, cuyo blanco de acción siempre es el SNC. Luego de haber infectado las células intestinales, se liberan, y atraviesan la barrera hematoencefálica, infectando astrocitos y neuronas. A pesar de los pocos estudios, y la poca claridad que hay en los que aceptan la infección asintomática de esta entidad, si se ha observado modificación del comportamiento en animales, sin embargo, no se ha visto daño en funciones ejecutivas en los sujetos infectados (Gajewski et al., 2014).

Por su parte, la larva Toxocara tiene la habilidad de invadir el cerebro humano provocando Toxocariasis. El contacto con perros portadores de T. Canis, puede infectar a un ser humano. Esta larva puede viajar por el torrente sanguíneo y subsecuentemente infectar organos como el hígado, corazón, pulmones, riñones, cerebro, músculos y ojos; y aunque raramente este tipo de neuroinfección produce sintomatología neurocognitiva, si se conoció un caso en Alemania de una mujer que presentó signos cognitivos y síntomas depresivos. No obstante, permanecen muchas sombras al respecto de la relación hospedero / parásito, que no explican la dishabilidad mental, epilepsia y otros desórdenes del SNC (Fan et al., 2015). 


\section{Evaluación}

Debido al escaso número de trabajos que analizan procesos cognitivos en niños, se ha tomado la edad entre 6 y 7 años como momento importante para ello, gracias a que en este periodo se inicia el aprendizaje de procesos relevantes como la lectura, la escritura y el cálculo matemático, cruciales a la hora de detectar alteración cognitiva que pudiese afectar la interacción en entornos académicos o sociales (Megías et al., 2015); para ello se ha hecho uso de instrumentos con un fuerte soporte teórico y organizados de manera sistémica que podrían facilitar la decisión para la elección del instrumento y del momento oportuno para ser usado (Vieira, Marques y Rizzo, 2015).

De igual manera, los entornos de evaluación de procesos cognitivos y afectivos, poseen una pureza muy superior a la que se ofrece en otros entornos donde se controlan menos otras variables intervinientes para lograr mayor ajuste del estímulo que se desea; no obstante, parece ser que este tipo de sitios, no constituyen los ingredientes completos para una valoración mas ecológica que podría obtenerse, paradógicamente, en un consultorio, sin aparatos de monitorización, pero con la supervisión y seguimiento del profesional entrenado (Marino et al., 2017). En este sentido, la valoración neuropsicológica de las funciones cognitivas sigue siendo un reto a nivel profesional e investigativo, las baterías de funciones ejecutivas proveen alta confiabilidad y validez de dichos procesos. "Debido a que la corteza prefrontal es la estructura cerebral que más se tarda en alcanzar su neurodesarollo, tiene mayor sensibilidad a las condiciones ambientales enriquecedoras pero también negativas como toxinas y estresores ambientales" (Flores, Ostrosky-Solís y Lozano, 2008), que pueden evidenciarse en muchas de estas pruebas (Chimbo Ch, 2014; Gajewski et al., 2014; Nimgaonkar et al., 2016; Silveira, 2016).

Otras formas de evaluación del funcionamiento cerebral y que tienen orientación hacia procesos cognitivos y afectivos, son las neuroimágenes por medio de Resonancia Magnética, Eye tracker, Registros psicofisiológicos y Evaluaciones conductuales (Marino et al., 2017). Así las cosas, los hallazgos que se van obteniendo mediante estas técnicas imagenológicas permite la comprensión de ciertas facetas y estructuras de procesos cognitivos como los diferentes tipos de memoria y su capacidad sustancial por definición (codificación y recuperación) (Megías et al., 2015).

El electroencefalograma, por su parte, permite detectar cambios en el potencial eléctrico, la Tomografía por Emisión de Positrones PET, y la Resonancia Magnética Funcional MRIf, a través del flujo sanguíneo de una zona cerebral específica, permiten detectar la actividad neuronal de la región implicada. Y 
no menos importante, es la confluencia interdisciplinar para el estudio de la mente en el gran marco de la ciencia cognitiva y neurociencias (Rivas, 2008).

\section{Tratamiento}

Debido a que algunas infecciones del SNC y otras encefalopatías no han sido muy estudiadas y en consecuencia poco entendidas, se ha perdido la clave para la generación de elementos y actividades neuroprotectoras con lo cual también la posibilidad de soporte para aquellos sobrevivientes de daños cerebrales por infección y/o sepsis (Widmann y Heneka, 2014). De igual manera, individuos con deterioro cognitivo podrían requerir mas intervenciones centradas en las consecuencias específicas de sus debilidades neurocognitivas y los factores de riesgo que contribuyen a ese deterioro (Gicas et al., 2014). Por ejemplo, el ejercicio aeróbico, ha demostrado utilidad y eficacia en cambios cognitivos asociados a la marcha de sujetos afectados solo despúes de la actividad física (Silveira, 2016). Por último, el tratamiento con antimicrobianos ha sido diseñado para limpiar la infección o disminuir la carga infecciosa, disminuir la severidad de la enfermedad y de alguna manera proveer cierto grado de prevención secundaria contra los efectos de la infección en el SNC. Para infecciones virales, a exepción de HSV y HIV, existen a menudo tratamientos no específicos, es decir, con antimicrobianos y no con antivirales o antirretrovirales que ataque directamente al virus causante de la infección (John et al., 2015).

\section{Conclusiones}

Los procesos cognitivos constituyen en esencia ser capaz de construir significados desde los diferentes aspectos y manifestaciones del mundo y la vida (Mariángel y Jiménez, 2015; Rivas, 2008) para modificar el proceso de aprendizaje y autorregularse con la ayuda de instrumentos culturales y de distintas áreas del lóbulo frontal, sobre todo para memoria, lenguaje y función ejecutiva. Sin embargo, la misma fragilidad humana nos hace proclives, no solo al deterioro normal por envejecimiento, sino al provocado por etiologías infecciosas virales, bacterianas, parasitarias y fúngicas.

El deterioro cognitivo provocado por agentes infecciosos, puede ser tan variable como la fuente del ataque mismo, con lo cual las consecuencias se ligan mucho a la susceptibilidad de las células cerebrales ante el agente estresor, de la capacidad del hospedero para defenderse, del tiempo prodrómico de la infección, del tiempo en que tardan los síntomas para aparecer, de la posibilidad de recibir una atención adecuada y oportuna y de la posibilidad para 
recibir manejo preventivo y rehabilitatorio. Con lo cual, conocer la respuesta cerebral y, por ende, la manifestación de los procesos cognitivos ante infecciones, permite al clínico anticipar daños y trabajar de manera preventiva y multidisciplinaria a favor de la preservación de procesos mentales por medio de la generación de elementos y actividades neuroprotectoras, que de otra manera resultarían deteriorados dejando secuelas importantes a nivel individual y del entorno social, familiar, académico y laboral de los sujetos afectados.

En este orden de ideas, se hace importante poder discernir el cuidado de las diferentes funciones cognitivas, tanto desde prácticas de estilos de vida saludable o prevención primaria (Portellano, 2005), como desde el uso de la tecnología computarizada que permite la optimización de la valoración neuropsicológica para las funciones cognitivas.

\section{Referencias}

Ackermann, C., van Toorn, R., Andronikou, S. (2019). Human immunodeficiency virus-related cerebral white matter disease in children. Pediatric Radiology, 49(5), 652-662. https://doi.org/10.1007/s00247-018-4310-x

Aguado, L. (2002). Procesos cognitivos y sistemas cerebrales de la emoción. Revista de Neurologia, 34(12), 1161-1170.

Cabello, J. B. (2003). Programa de habilidades en lectura crítica CASPe. Entendiendo la evidencia sobre la eficacia clínica. In CASPe. Guías CASPe de Lectura Crítica de la Literatura Médica. (p. 5). Alicante. Retrieved from http://www.redcaspe.org

Carmilema T, J. C., Fajardo B, M. F. (2018). Evaluación sobre los niveles de atención y memoria en pacientes drogodependientes en condición de internamiento. Universidad de Cuenca. https://doi.org/10.22201/fq.18708404e.2004.3.66178

Chimbo Ch, E. W. (2014). La drogodependencia y su influencia en la capacidad para la toma de decisiones. Universidad Central Del Ecuador. Universidad Central del Ecuador.

Cohen, S., Ter Stege, J. A., Geurtsen, G. J., Scherpbier, H. J., Kuijpers, T. W., Reiss, P., ... Pajkrt, D. (2015). Poorer cognitive performance in perinatally HIV-infected children versus healthy socioeconomically matched controls. Clinical Infectious Diseases, 60(7), 1111-1119. https://doi.org/10.1093/cid/ciu1144

Fan, C. K., Holland, C. V., Loxton, K., Barghouth, U. (2015). Cerebral toxocariasis: Silent progression to neurodegenerative disorders? Clinical Microbiology Reviews, 28(3), 663-686. https://doi.org/10.1128/CMR.00106-14 
Fernández, G., Orozco, D., Agamennoni, O. (s.f.). Evaluación Cognitiva mediante Eye tracking ( ECEt). Buenos Aires, Argentina: Universidad Nacional del Sur. http://www.gmics.diec.uns.edu.ar/images/PDFs/ EvaluacinCognitivaMedianteEyetrackingFolletoMedico.pdf

Ferrari, J., Campos, G., Kalil, J., Nitrini, R., Jacob-Filho, W., Tesseroli, J., D.T.Siqueira, S. (2016). Oral Infections and Cytokine Levels in Patients with Alzheimer's Disease and Mild Cognitive Impairment Compared with Controls. Journal of Alzheimer's Disease, 52(4), 1479-1485. https://doi.org/10.3233/JAD-160212

Flores, J., Ostrosky-Solís, F., Lozano, A. (2008). Batería de Funciones Frontales y Ejecutivas: presentacion. Revista de Neuropsicología, neuropsiquaiatría y neurociencias, 8 (1), 141-158, https://aalfredoardila.files.wordpress. com/2013/07/ardila-a-ed-2008-funciones-ejecutivas-neuropsicologianeuropsiquiatria-y-neurociencias-vol-8-n1.pdf

Gajewski, P. D., Falkenstein, M., Hengstler, J. G., Golka, K. (2014). Toxoplasma gondii impairs memory in infected seniors. Brain, Behavior, and Immunity, 36, 193-199. https://doi.org/10.1016/j.bbi.2013.11.019

García-Herranz, S., Díaz-Mardomingo, M., Peraita, H. (2014). Evaluación y seguimiento del envejecimiento sano y con deterioro cognitivo leve (DCL) a través del TAVEC. Anales de Psicología, 30(1), 373-380. https://doi.org/10.6018/ analesps.30.1.150711

Gicas, K. M., Vila-Rodriguez, F., Paquet, K., Barr, A. M., Procyshyn, R. M., Lang, D. J., ... Thornton, A. E. (2014). Neurocognitive profiles of marginally housed persons with comorbid substance dependence, viral infection, and psychiatric illness. Journal of Clinical and Experimental Neuropsychology, 36(10), 1009-1022. https://doi.org/10.1080/13803395.2014.963519

Golub, M. D., Chase, S. M., Batista, A. P., Yu, B. M. (2016). Brain-computer interfaces for dissecting cognitive processes underlying sensorimotor control. Current Opinion in Neurobiology, 37, 53-58. https://doi.org/10.1016/j.conb.2015.12.005

Guzmán de R, C. (2013). Evaluación Neuropsicológica de los Procesos Cognitivos en la Epilepsia. Red de Revistas Científicas de América Latina y El Caribe, España y Portugal, 11(2), 193-227. Retrieved from http://www.redalyc.org/ html/4615/461545460005/

Hagler, S., Brugge, H., Pavel, M. (2014). Assessing executive function using a computer game: computational modeling of cognitive processes. IEEE Journal of Biomedical and Health Informatics, 18(4), 1442-1452. https://doi.org/10.1109/ JBHI.2014.2299793 
Hamdani, N., Daban-Huard, C., Lajnef, M., Gadel, R., Le Corvoisier, P., Delavest, M., ... Leboyer, M. (2015). Cognitive deterioration among bipolar disorder patients infected by Toxoplasma gondii is correlated to interleukin 6 levels. Journal of Affective Disorders, 179, 161-166. https://doi.org/10.1016/j.jad.2015.03.038

Hung, T. H., Chen, V. C. H., Yang, Y. H., Tsai, C. S., Lu, M. L., McIntyre, R. S., ... Huang, K. Y. (2018). Association between enterovirus infection and speech and language impairments: A nationwide population-based study. Research in Developmental Disabilities, 77, 76-86. https://doi.org/10.1016/j.ridd.2018.04.017

Introzzi, I., Canet-Juric, L., Montes, S., López, S., Mascarello, G. (2015). Procesos Inhibitorios y flexibilidad cognitiva : evidencia a favor de la Teoría de la Inercia Atencional. International Journal of Psychology Research, 8(2), 61-75. http:// www.scielo.org.co/pdf/ijpr/v8n2/v8n2a06.pdf

John, C. C., Carabin, H., Montano, S. M., Bangirana, P., Zunt, J. R., Peterson, P. K. (2015). Global research priorities for infections that affect the nervous system. Nature, 527(7578):S178-86. doi: 10.1038/nature16033.

Karachaliou, M., Chatzi, L., Roumeliotaki, T., Kampouri, M., Kyriklaki, A., Koutra, K., ... de Sanjose, S. (2016). Common infections with polyomaviruses and herpesviruses and neuropsychological development at 4 years of age, the Rhea birth cohort in Crete, Greece. Journal of Child Psychology and Psychiatry and Allied Disciplines, 57(11), 1268-1276. https://doi.org/10.1111/jcpp.12582

Lattanzi, S., Luzzi, S., Provinciali, L., Silvestrini, M. (2014). Blood pressure variability predicts cognitive decline in Alzheimer's disease patients. Neurobiology of Aging, 35(10), 2282-2287. https://doi.org/10.1016/j.neurobiolaging.2014.04.023

Licastro, F., Raschi, E., Carbone, I., Porcellini, E., Caruso, C. (2015). Variants in antiviral genes are risk factors for cognitive decline and dementia. Journal of Alzheimer's Disease, 46(3), 655-663. https://doi.org/10.3233/JAD-142718

Llinás, R. (2003). El cerebro y el mito del Yo. El papel de las neuronas en el pensamiento y el comportamiento humanos. Bogotá, Colombia: Editorial Norma.

Luna-Lario, P., Azcárate-Jiménez, L., Seijas-Gómez, R., Tirapu-Ustárroz, J. (2015). Propuesta de una batería neuropsicológica de evaluación cognitiva para detectar y discriminar deterioro cognitivo leve y demencias. Revista de Neurologia, 60(12), 553-561. Retrieved from http://www.ncbi.nlm.nih.gov/pubmed/26062828

Macbeth, G., Cortada, N., Razumiejeczyk, E., López, A. (2008). Eficacia del monitoreo metacognitivo en procesos de atribución de verdad, falsedad y novedad. Signos Universitarios. 27(43), 63-76, Retrieved from http://p3.usal.edu.ar/index.php/ signos/article/view/2163/2710 
Mariángel, S. V., Jiménez, J. E. (2015). Desarrollo de la conciencia sintáctica y fonológica en niños chilenos: un estudio transversal. Revista Latinoamericana de Psicología, 48(1), 1-7. https://doi.org/10.1016/j.rlp.2015.09.010

Marino, J., Jaldo, R., Arias, J., Palma, M. (2017). Neurociencia de las capacidades y los procesos cognitivos. Buenos Aires: Editoral brujas.

Marmor, M., Glickman, L., Shofer, F., Faich, L. A., Rosenberg, C., Cornblatt, B., Friedman, S. (1987). Toxocara canis infection of children: Epidemiologic and neuropsychologic findings. American Journal of Public Health, 77(5), 554-559. https://doi.org/10.2105/AJPH.77.5.554

Megías, M., Esteban, L., Roldán, M. D., Estévez, Á., Sanchez-Joya, M., Ramos-Lizana, J. (2015). Evaluación neuropsicológica de procesos cognitivos en niños de siete años de edad nacidos pretérmino. Anales de Psicologia, 31(3), 1052-1061. http:// dx.doi.org/10.6018/analesps.32.1.151881

Mias, C. D., Bastida, M. F., Del Boca, M. L., Legeren, A. L. (2017). Edades críticas en el declive de distintas funciones neurocognitivas en adultos jóvenes y mayores. Anuario de Investigaciones, 3(2), 182-190. https://revistas.unc.edu.ar/index.php/ aifp/article/view/18829

Moreno, C., Castelblanco, I., Peña, K., Sandoval, M., Zamudio, J., Prieto, A. (2016). Efectos fisiológicos de Cannabis Sativa en universitarios consumidores. (R. Carvajalino, N. De la Cuadra, M. Jaramillo, M. de Olivos, Eds.) (Primera). Bogotá, Colombia: Editorial UD.

Nelson, S., Greene, T., Ernhart, C. B. (1996). Toxocara canis infection in preschool age children: Risk factors and the cognitive development of preschool children. Neurotoxicology and Teratology, 18(2), 167-174. https://doi.org/10.1016/08920362(95)02018-7

Nimgaonkar, V., Yolken, R., Wang, T., Chung-Chou, H., McClain, L., McDade, E., ... Ganguli, M. (2016). Temporal cognitive decline associated with exposure to infectious agents in a population-based, aging cohort. Alzheimer Disease and Associated Disorders, 00(0), 216-222. Retrieved from http://www.ncbi.nlm.nih. gov/pmc/articles/PMC4919236/

Ollari, J. (1999). Declinación cognitiva y deterioro cognitivo. Envejecimiento normal y enfermedades de la senescencia. Rev Neurol Arg, 23(1), 3-8, http:// www.imbiomed.com.mx/1/1/articulos.php? method=showDetail\&id_ articulo $=16089 \&$ \&id_seccion $=1316 \&$ id_ejemplar $=1666 \& i d$ revista $=95$

Portellano, J. A. (2005). Introducción a la neuropsicología. (J. M. Cejudo, Ed.), Mc Graw Hill. Madrid, España: Mc Graw-Hill. 
Ragin, A. B., Wu, Y., Gao, Y., Keating, S., Du, H., Sammet, C., ... Epstein, L. G. (2015). Brain alterations within the first 100 days of HIV infection. Annals of Clinical and Translational Neurology, 2(1), 12-21. https://doi.org/10.1002/acn3.136

Ravindran, O. S., Rani, M., Priya, G. (2014). Cognitive deficits in HIV infected children. Indian Journal of Psychological Medicine, 36(3), 255. https://doi. org/10.4103/0253-7176.135373

Rivas N, M. (2008). Procesos cognitivos y aprendizaje significativo. Madrid, España: Subdirección General de Inspección Educativa de la Viceconsejería de Organización Educativa de la Comunidad de Madrid, http://www.deposoft.com.ar/repo/ publicaciones/A9R6652.pdf

Rojo-Mota, G., Pedrero-Perez, E., Ruiz-Sánchez, J., Llanero-Luque, M., Puerta-Garcia, C. (2013). Cribado neurocognitivo en adictos a sustancias: la evaluación cognitiva de Montreal. Revista de Neurologia, 56(3), 129-136. Retrieved from http://www. neurologia.com/pdf/Web/5603/bh030129.pdf\%5Cnhttp://ovidsp.ovid.com/ ovidweb.cgi? $\mathrm{T}=\mathrm{JS} \& \mathrm{PAGE}=$ reference $\& \mathrm{D}=$ emed $11 \& \mathrm{NEWS}=\mathrm{N} \& \mathrm{AN}=2013107433$

Sandoz, M., Démonet, J. F., Fossard, M. (2014). Theory of mind and cognitive processes in aging and Alzheimer type dementia: A systematic review. Aging and Mental Health, 18(7), 815-827. https://doi.org/10.1080/13607863.2014.899974

Silveira, C. (2016). The effects of physical exercise on cognition of individuals with Parkinson's disease. Doctoral thesis, University Of Waterloo, https://pdfs. semanticscholar.org/43f2/599a72fa1590fd24e30b2ba77a18160a7c2a.pdf

Solís-Marcos, I., Castellano-Guerrero, A., Domínguez Morales, R., León-Carrión, J. (2019). Predictores de la recuperación funcional cognitiva en pacientes con traumatismo craneoencefálico. Revista de Neurología, 58(07), 296-302. https:// doi.org/10.33588/rn.5807.2013514

Spagnolo, F., Scomazzoni, F., Fichera, M., Comi, G., Volontè, M. A. (2014). Secondary cervical dystonic tremor after Japanese encephalitis. Neurological Sciences, 35, 491-493. https://doi.org/10.1007/s10072-013-1579-2

Su, T., Caan, M. W. A., Wit, F. W. N. M., Schouten, J., Geurtsen, G. J., Cole, J. H., ... Majoie, C. B. (2016). White matter structure alterations in HIV-1-infected men with sustained suppression of viraemia on treatment. Aids, 30(2), 311-322. https://doi.org/10.1097/QAD.0000000000000945

Takeda, S., Sato, N., Morishita, R. (2014). Systemic inflammation, blood-brain barrier vulnerability and cognitive / non-cognitive symptoms in Alzheimer disease: Relevance to pathogenesis and therapy. Frontiers in Aging Neuroscience, 6, 1-8. https://doi.org/10.3389/fnagi.2014.00171 
Vergel C, R. (2014). El signo en Vygotski y su vínculo con el desarrollo de los procesos psicológicos superiores. Folios, (39), 65-76. https://doi. org/10.17227/01234870.39folios65.76

Vieira, V., Marques, F., Rizzo, A. (2015). Learning Difficulties in Computing Courses : Cognitive Processes Assessment Methods Research and Application. XI Brazilian Symposium on Information System, 31-38. https://doi.org/10.13140/ RG.2.1.4560.1768

Villalba, S., Tortajada, R. (2014).Estimulación cognitiva: una revisión neuropsicológica. Therapeía 6, (6), 73-93. http://gamisolution.es/wp-content/uploads/2016/06/ Dialnet-EstimulacionCognitivaUnaRevisionNeuropsicologica-5149523.pdf

Widmann, C. N., Heneka, M. T. (2014). Long-term cerebral consequences of sepsis. The Lancet Neurology, 13, 630-636. https://doi.org/10.1016/S1474-4422(14)70017-1

Wu, Y. H., Cui, X. Y., Yang, W., Fan, D. Y., Liu, D., Wang, P. G., An, J. (2018). Zika Virus Infection in Hypothalamus Causes Hormone Deficiencies and Leads to Irreversible Growth Delay and Memory Impairment in Mice. Cell Reports, 25(4), 1537-1547. https://doi.org/10.1016/j.celrep.2018.10.025

Zhao, L., Zhou, M., Wang, B., Guo, J., Chen, N., He, L. (2015). Clinical characteristics and outcome of clinically diagnosed viral encephalitis in southwest China. Neurological Sciences, 36, 2191-2197. https://doi.org/10.1007/s10072-0152333-8 